
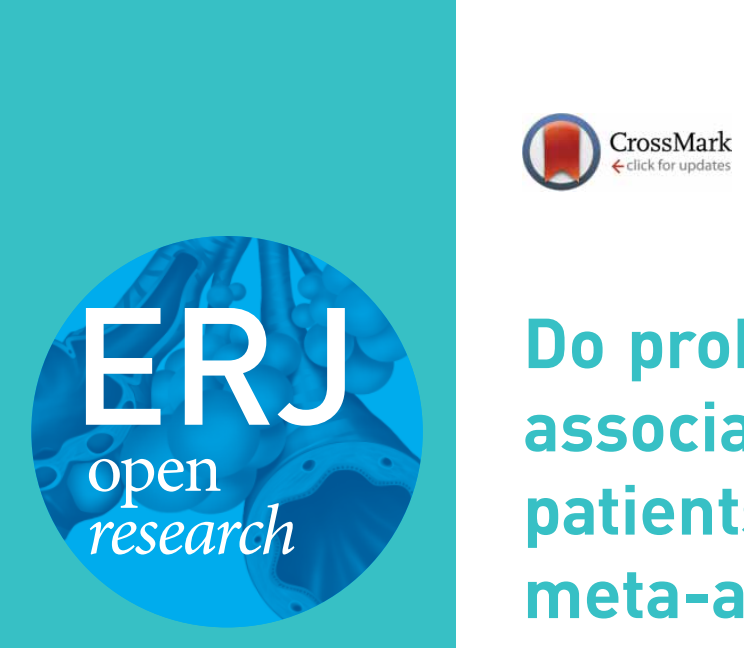

\title{
Do probiotics help prevent ventilator- associated pneumonia in critically ill patients? A systematic review with meta-analysis
}

\author{
Jie Zhao, Lei-qing Li, Cheng-yang Chen, Gen-sheng Zhang, Wei Cui and \\ Bao-ping Tian
}

Affiliation: Dept of Critical Care Medicine, The Second Affiliated Hospital, Zhejiang University School of Medicine, Hangzhou, Zhejiang, 310009, China.

Correspondence: Bao-ping Tian, Dept of Critical Care Medicine, The Second Affiliated Hospital, Zhejiang University School of Medicine, 88 Jiefang Rd, Hangzhou 310009, China. E-mail: TianBPAzju.edu.cn

\section{ABSTRACT}

Background: Probiotic treatments might contribute to the prevention of ventilator-associated pneumonia (VAP). Due to its unclear clinical effects, here we intend to assess the preventive effect and safety of probiotics on intensive care unit (ICU) patients.

Methods: Eligible randomised controlled trials were selected in databases until 30 September 2019. The characteristics of the studies were extracted, including study design, definition of VAP, probiotics intervention, category of included patients, incidence of VAP, mortality, duration of mechanical ventilation $(\mathrm{MV})$ and ICU stay. Heterogeneity was evaluated by Chi-squared and $\mathrm{I}^{2}$ tests.

Results: 15 studies involving 2039 patients were identified for analysis. The pooled analysis suggests significant reduction on VAP (risk ratio, $0.68 ; 95 \% \mathrm{Cl}, 0.60$ to $0.77 ; \mathrm{p}<0.00001$ ) in a fixed-effects model. Subgroup analyses performed on the category of clinical and microbiological criteria both support the above conclusion; however, there were no significant differences in duration of MV or length of ICU stay in a random-effects model. Also, no significant differences in total mortality, overall mortality, 28-day mortality or 90-day mortality were found in the fixed-effects model.

Conclusions: The probiotics helped to prevent VAP without impacting the duration of MV, length of ICU stay or mortality.

@ERSpublications

Pooled analysis suggests significant reduction in VAP in probiotic-treated patients but no change in the length of ICU stay, duration of mechanical ventilation or mortality https://bit.ly/30FfDTD

Cite this article as: Zhao J, Li L-qing, Chen C-yang, et al. Do probiotics help prevent ventilatorassociated pneumonia in critically ill patients? A systematic review with meta-analysis. ERJ Open Res 2021; 7: 00302-2020 [https://doi.org/10.1183/23120541.00302-2020].

This article has supplementary material available from openres.ersjournals.com

Received: 24 May 2020 | Accepted after revision: 28 Sept 2020

Copyright $\odot$ ERS 2021. This article is open access and distributed under the terms of the Creative Commons Attribution Non-Commercial Licence 4.0. 


\section{Background}

Ventilator-associated pneumonia (VAP) is a nosocomial infection characterised by onset after $48 \mathrm{~h}$ of the application of mechanical ventilation (MV) [1]. VAP is among the most common infections in hospitalised patients and affects between $9 \%$ and $27 \%$ of intubated patients [2]. VAP remains a serious problem in the intensive care unit (ICU), which has considerable consequences, including significant morbidity, mortality, markedly prolonged ICU length of stay, and increased ventilator days [3-5]. Patients who develop VAP cost US $\$ 40000$ more to treat than those who do not [6]. Currently, initial appropriate antimicrobial therapy with a length of 7 or 8 days for most patients is a critical strategy of VAP treatment. However, multidrug-resistant (MDR) or extensive-drug resistant (XDR) pathogens are becoming prevalent as antibiotic drugs continue to be used inappropriately, which means our current antibiotic strategies are becoming ineffective [7]. Some scientists have now devoted themselves to developing a new generation of synthetic antimicrobials, aiming to minimise the risks of spontaneous resistance and toxicity associated with currently available antibiotics [8]. The major cause of VAP is aspiration of either microorganism from the oropharynx or fragments of biofilms from the endotracheal tube, and inhibition of the colonisation and formation of the biofilm are viewed as a feasible strategy $[9,10]$. Probiotics are living microorganisms and considered as an option for the prevention of VAP through minimising the colonisation by virulent species or modulating the host immune defence [11]. The conclusions of randomised controlled trials (RCTs) conducted in succession are inconsistent. Meta-analysis performed by SiEmpos et al. [12], Bo et al. [13], vAN RUISSEN et al. [14] and Su et al. [15] supported the efficacy of probiotics in prevention of VAP, while other meta-analyses conducted by Gu et al. [16] and WANG et al. [17] denied the positive effect of probiotics. Due to the limited number of studies, low quality of the evidence and the wide confidence interval $(\mathrm{Cl})$ of the estimated effect, these meta-analyses cannot provide sufficient evidence to draw a conclusion supporting the efficacy of probiotics in preventing VAP. We further standardised the inclusion criteria with the objective of improving the quality of evidence. For example, we excluded the RCTs conducted by KotZAMPASsi et al. [18], which just illustrated the reduction of respiratory tract infection without a define of VAP. Then, we performed a meta-analysis after including the most recent RCTs and present results in order to summarise the effect and limitation of probiotics for preventing VAP in the critically ill.

\section{Methods}

\section{Search strategies for data}

This systematic meta-analysis was conducted according to the Cochrane Handbook for Systematic Reviews of Interventions [19], and the International prospective register of systematic reviews (PROSPERO) [20]. Although it was not registered, the entire process was in accordance with the above requirements. The protocol has not been previously published. All of the articles reporting the prophylactic effect of probiotics for VAP in critically ill patients were systematically retrieved through databases, including PubMed, Medline, EMBASE, the Cochrane Controlled Trials Register databases, and the China National Knowledge Infrastructure database until 30 September 2019. The database search was conducted with the following Medical Subject Headings entry terms and thesaurus vocabulary for indexing articles: "probiotic", "probiotics”, "prebiotic", "prebiotics", "symbiotic”, “symbiotics”, "pneumonia”, "pneumonias”, "ventilator", "ventilation", mechanical ventilation", "ventilator-associated pneumonia”, "random”, "randomised", "control", "controlled", "trial", "trials", "clinical trial”, "clinical trials", "randomised controlled trial", "randomised controlled trials", "RCT", "RCTs". No language restriction was applied. To identify other potentially eligible articles, studies were searched manually on primary researches, review articles, and manufacturers' websites for trial information by reviewing titles, abstracts, and full texts.

\section{Inclusion and exclusion criteria}

Eligible articles were selected according to the Cochrane Handbook for Systematic Reviews of Interventions and the PICOS model for the definition of inclusion criteria [19]: 1) population: all of the participants had an expected need of MV for at least $48 \mathrm{~h}$; 2) intervention: any type of probiotics regimen; 3) comparison: with placebo or other therapies; 4) outcomes: incidence of VAP, mortality within 28 days, ICU and hospital length of stay, duration of MV, complications, number of free days of antibiotic use for VAP at day 28; and 5) study design: RCTs. However, probiotic formulation and dose or time of administration, minimum sample size, and age of participators were not included as criteria. Studies were excluded if they had any of the following characteristics: 1) text without data about participant characteristics or outcome, such as comment, guideline, case, poster, and review; 2) studies were not performed in humans, or conducted in ex vivo cells or animals; 3) studies only analysed participants with a special occupation; 4) studies involving patients already with evidence of previous history of pneumonia; and 5) studies involving patients with immunosuppression. The eligible articles were judged and selected by two researchers independently. 
Data extraction and risk of bias assessment

Two investigators extracted the following information independently in related articles: study design, general characteristics of patients (sample size, age, number of females or males in each trial, sex, baseline of general condition, definition of VAP, therapeutic dosages and process of probiotics, the administration on ICU patients in placebo groups), and the primary or secondary outcomes, including the incidence of VAP, infectious complications, the proportions of eradication of colonisation, ICU, and hospital length of stay, duration of MV, mortality, faecal bacterial counts, organic acid concentration, length of antibiotic use for VAP, prevalence of microorganisms in surveillance cultures, and adverse events, among others. Some data were calculated with available data by Review Manager (RevMan, version 5.3.0., Cochrane Collaboration, Oxford, UK) if they were not provided directly in texts. Any discrepancies were resolved by a third reviewer after assessing the original literature. Heterogeneity among the included trials was assessed via the Chi-squared test and quantitatively measured through the $\mathrm{I}^{2}$ statistic. In situations of significant heterogeneity, the source was explored through sensitivity analysis. Subgroup analyses were made to evaluate the effect of probiotics on short-term mortality and long-term mortality. The risk of bias for the included papers was assessed using the Cochrane Handbook for Systematic Reviews of Interventions criteria [19]. A different definition of VAP for trial enrolment is an important source of heterogeneity and bias. Subanalysis was performed on the clinically confirmed VAP, quantitative microbiological confirmed VAP and nonquantitative microbiological confirmed VAP. The evaluation criteria were based on sample selection, allocation concealment (selection bias), blinding of participants and personnel (performance bias), incomplete outcome data (attrition bias), statistical analysis and outcome validation, selective reporting and free of source of funding (reporting bias) measured the degree of bias, definition of inclusion and exclusion criteria. They were categorised as low risk, high risk, and unclear risk. Funnel plots were used to identify possible instances of publication bias or other bias.

\section{Statistical analysis}

The incidence of VAP in patients exposed to probiotics, duration of MV, length of ICU stay, and mortality was analysed in this systematic review. For continuous variables, we calculated the standardised mean difference (SMD) with 95\% CI. The dichotomous data variables were measured by risk ratio and corresponding $95 \% \mathrm{CI}$. The p-value for the comparison between the groups was calculated, and a p-value $\leqslant 0.05$ was considered as statistically significant. Heterogeneity was considered significantly statistical $\left(\mathrm{p}<0.10\right.$ or $\left.\mathrm{I}^{2} \geqslant 50 \%\right)$ evaluated by Chi-squared and $\mathrm{I}^{2}$ tests in a fixed-effects model or random-effects model. Publication bias was assessed by visually inspecting funnel plots in this meta-analysis. The comparison of the outcome between the probiotic and placebo was conducted using Review Manager 5.3 (Revman, The Cochrane Collaboration, Oxford, UK) [19]. P-values $<0.05$ were considered statistically significant.

\section{Results}

Characteristics and risk of bias assessment of the included studies

This systematic analysis was conducted according to PROSPERO, and the PRISMA checklist is provided in supplemental table 1. A total of 342 studies were selected by searching PubMed, Medline, EMBASE, the Cochrane Database and the China National Knowledge Infrastructure database, and 29 publications remained after removing duplicated texts and excluding inappropriate articles (identified as a comment, guideline, case, poster, review, abstract without data, editorials, erratum via reviewing the titles and abstracts). As shown in figure 1, after full-text reviewing of the selected 29 articles, 15 studies with 2039 participants were identified for quantitative analysis and the systematic review. All of the studies were designed as RCTs, among which 13 trials were carried out on adults $[9,21-32]$ (age $\geqslant 16$ years), and two studies were conducted on children [33,34]. Only one article did not mention the detailed blind design [33]. Seven studies and two studies were designed as double-blind trials [23, 26-29, 31, 32] and single-blind trials $[22,24]$, respectively, while five studies were open-label trials $[9,21,25,30,34]$. All of the articles made the statistical analysis of the occurrence of VAP in patients, although the works by SHIMIzu et al. [24] did not provide definite details about diagnosis criteria of VAP [31]. The characteristics of the trials are classified in table 1 and supplemental table 2 . The risk of bias assessment of each included trial is displayed in figure 2. Briefly, the bias of analysis mostly originated from the bias of performance and outcome assessment among the included trials, in which only five studies showed low risk [23, 27, 28, 31, 32]. The studies conducted by Knight et al. [27] and Mahmoodpoor et al. [32] can be viewed as high-quality studies, which show low risk in every procedure of the clinical trial.

\section{Probiotics decreased the incidence of VAP}

The pooled analysis with a fixed-effects model suggested significantly decreased morbidity of VAP in the probiotics group compared with the placebo group (risk ratio, $0.68 ; 95 \% \mathrm{Cl}, 0.60$ to $0.77 ; \mathrm{p}<0.00001$ ) based on 15 studies involving 2039 patients [9, 21-34]. Chi-squared and $\mathrm{I}^{2}$ tests were used to evaluate the 
FIGURE 1 Outline of literature strategy, yielding 15 randomised publications that compared probiotics with conventional treatment strategies.
Records identified through database searching

(PubMed, Medline, EMBASE, Cochrane, CNKI) $n=342$

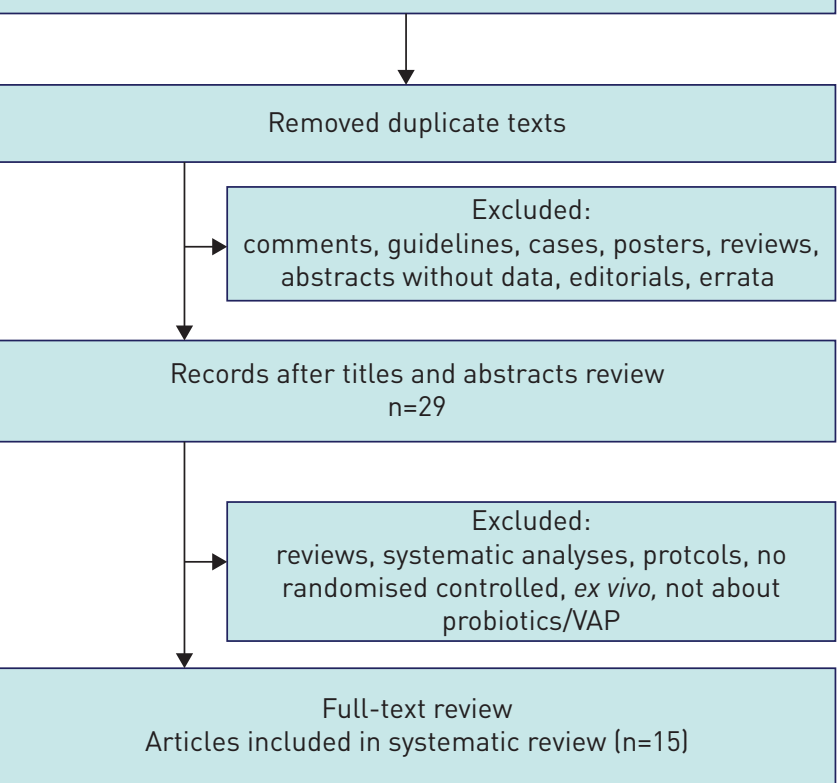

statistical heterogeneity of these studies. As shown in figure 3, mild statistical heterogeneity was observed ( $\mathrm{p}=0.08, \mathrm{I}^{2}=36 \%$ ). However, among the included trials, eight studies did not detect a significant preventive effect for probiotics compared to a control group [9, 21, 22, 26-30]. The sample size was too small to detect minor discrepancy between groups, which might account for the two trails reporting a different result $[9,22]$. OudHuIs et al. [35] reported that the effect of probiotics in preventing VAP was not superior to the selective decontamination of the digestive tract (SDD) $(p=0.25)$, while isolates of antibiotic resistance strains were significantly higher in the probiotics group than in the SDD group $(\mathrm{p}<0.05)[36]$. ForESTIER et al. [29] observed a decreased VAP frequency but without reaching statistical significance $(\mathrm{p}>0.05)$, while VAP occurrence was significantly delayed in the probiotics group (11 days versus 50 days, $\mathrm{p}=0.01$ ). The absence of probiotics treatment (adjusted hazard ratio, 3.2; 95\% CI, 1.1 to 9.1) was found to be an independent factor associated with increased risk for Pseudomonas aeruginosa respiratory infection [29]. Rongrungruang et al. [21] carried out their trial with a basic oral administration of chlorhexidine $(\mathrm{CHX})$ for all the patients and found no significant difference in VAP frequency $(\mathrm{p}=0.46)[37,38]$. However, we cannot ignore the interfering impact of CHX, which might impair the viability of the probiotics. Interestingly, KLARIN et al. $[9,30]$ performed an RCT comparing oral application of probiotics and CHX in 2008 and subsequently continued the study after expanding the sample size 10 years later. Both trials found that probiotics were not superior to CHX ( $p>0.05)$ [37-39]. Giamarellos-Bourboulis et al. [26] have reported that probiotics did not help to prevent VAP with diverse pathogens ( $p>0.05)$, but contributed to the reduction of VAP of Acinetobacter baumanii ( $\mathrm{p}=0.047)$, which suggests that the effects of probiotics depend on the strain of bacteria. A subgroup analysis was performed based on category of diagnostic criteria of VAP, which was reported to have associations with VAP occurrence, duration of ICUs and mortality [40]. The subgroup analysis of clinically confirmed VAP (risk ratio, 0.69; 95\% $\mathrm{Cl}, 0.55$ to $0.86 ; \mathrm{p}=0.001$ ) and quantitative microbiological confirmed VAP (risk ratio, $0.80 ; 95 \% \mathrm{Cl}, 0.66$ to 0.97 ; $\mathrm{p}=0.02$ ) and nonquantitative microbiological confirmed VAP (risk ratio, $0.63 ; 95 \% \mathrm{Cl}, 0.47$ to 0.83 ; $\mathrm{p}=0.001$ ) all suggested a reduction of VAP incidence (figure 3 ). Sensitivity analysis by removing each trial also showed a significant reduction of VAP incidence similarly to the overall analysis. The funnel plot analysis was seemingly symmetric, suggesting no publication bias (supplemental figure 1).

\section{Probiotics did not affect the duration of mechanical ventilation}

Comprehensive analysis based on the eight studies involving 1200 participators that evaluated duration of MV indicated that there was no significant reduction in the probiotic group compared to the control group (SMD, $-0.20 ; 95 \% \mathrm{Cl},-0.41$ to $0.01 ; \mathrm{p}=0.07$ ) in a random-effects model (supplemental figure 2 ) $[9,23,25,27,28$, $31,32,34]$. The results were limited by high heterogeneity $\left(p=0.003 ; I^{2}=67 \%\right)$, indicating possible bias. The subgroup analysis of clinically confirmed VAP (SMD, $-0.11 ; 95 \% \mathrm{Cl},-0.53$ to $\left.0.31 ; \mathrm{p}=0.61 ; \mathrm{I}^{2}=80 \%\right)$, 
TABLE 1 Characteristics and designs of the included studies

\begin{tabular}{|c|c|c|c|c|c|c|c|c|}
\hline $\begin{array}{l}\text { First author year } \\
\text { [ref.] }\end{array}$ & Study design & Male/patients & Age years & Inclusion criteria & Definition of VAP & $\begin{array}{l}\text { Probiotics } \\
\text { intervention }\end{array}$ & Primary outcomes & Secondary outcomes \\
\hline $\begin{array}{l}\text { MAHMOODPOOR } 2019 \\
\text { [32] }\end{array}$ & $\begin{array}{l}\text { Prospective, } \\
\text { double-blind, } \\
\text { randomise, } \\
\text { controlled } \\
\text { trial }\end{array}$ & $55 / 102$ & $\begin{array}{l}\text { Probiotics: } \\
\text { 59.1 } \pm 12.9 / \\
\text { Control: } \\
57.5 \pm 14.5\end{array}$ & $\begin{array}{l}\text { Patients } \geqslant 18 \text { years old } \\
\text { and had been } \\
\text { undergoing } \mathrm{MV} \text { for } \\
>48 \mathrm{~h}\end{array}$ & $\begin{array}{l}\text { A new or persistent } \\
\text { infiltration on chest } \\
\text { radiography with } 2 \text { of } \\
\text { the } 3 \text { criteria of } 1) \\
\text { temperature }>38^{\circ} \mathrm{C} \text { or } \\
<36^{\circ} \mathrm{C} ; 2 \text { ) leukocytosis } \\
\text { or leukopenia; } 3 \text { ) } \\
\text { purulent sputum } \\
\text { underwent BAL } 4 \text { ) the } \\
\text { quantitative BAL } \\
\text { cultures had at least } \\
10^{4} \mathrm{CFU} \cdot \mathrm{mL}^{-1}\end{array}$ & $\begin{array}{l}\text { Lac+Bif+Str: } 1 \\
\text { capsule every } \\
12 \mathrm{~h} \text { daily for } \\
14 \text { days. Each } \\
\text { capsule } \\
\text { contained } 10^{10} \\
\text { bacteria } \\
\text { consisting of } \\
\text { Lactobacillus } \\
\text { species (casei, } \\
\text { acidophilus, } \\
\text { rhamnosus, } \\
\text { bulgaricus), } \\
\text { Bifidobacterium } \\
\text { species (breve, } \\
\text { longum) and } \\
\text { Streptococcus } \\
\text { thermophilus }\end{array}$ & VAP frequency & $\begin{array}{l}\text { ICU and hospital } \\
\text { length of stay, } \\
\text { duration of MV, } \\
\text { complications }\end{array}$ \\
\hline KLARIN 2018 [30] & $\begin{array}{l}\text { A multicentre, } \\
\text { prospective, } \\
\text { randomised } \\
\text { controlled } \\
\text { open trial }\end{array}$ & $76 / 137$ & $\begin{array}{l}\text { Probiotics: } \\
66 \text { (57-76)/ } \\
\text { Control: } \\
65.5 \text { (54-75) }\end{array}$ & $\begin{array}{l}\text { Patients } \geqslant 18 \text { years old, } \\
\text { critically ill with an } \\
\text { anticipated need for } \\
\text { MV of at least } 24 \mathrm{~h} \text {, not } \\
\text { moribund, not having } \\
\text { pneumonia, no } \\
\text { fractures in the facial } \\
\text { skeleton or the base of } \\
\text { the skull, no oral } \\
\text { ulcers, not immune } \\
\text { deficient, not a carrier } \\
\text { of HIV or viral hepatitis, } \\
\text { not being } \\
\text { tracheotomised, and no } \\
\text { standard oral care } \\
\text { before }\end{array}$ & $\begin{array}{l}\text { A new, persistent or } \\
\text { progressive infiltrate on } \\
\text { chest radiograph } \\
\text { combined with at least } \\
3 \text { of the other } 4 \text { criteria: } \\
\text { 1) a purulent tracheal } \\
\text { aspirate; } 2 \text { ) positive } \\
\text { culture of tracheal } \\
\text { aspirates occurring } \\
\text { after } 48 \mathrm{~h} \text { of } \mathrm{MV} ; 3) \\
\text { rectal or urine bladder } \\
\text { temperature }>38.0^{\circ} \mathrm{C} \text { or } \\
\left.<35.5^{\circ} \mathrm{C} ; 4\right) \\
\text { WBC }>12 \times 10^{3} \text { or } \\
<3 \times 10^{3} \cdot \mathrm{mm}^{-3} \text { or a rapid } \\
\text { increase in WBC count } \\
\text { without suspicion of } \\
\text { infection in another } \\
\text { organ }\end{array}$ & $\begin{array}{l}\text { Lac: Lp299 } \\
\text { [Lactobacillus } \\
\text { plantarum 299] } \\
\text { were applied to } \\
\text { the mucosal } \\
\text { surface of the } \\
\text { oral cavity twice a } \\
\text { day, the } \\
\text { subsequent } \\
\text { cleansing was } \\
\text { performed with } \\
\text { gauze swabs } \\
\text { soaked in } \\
\text { carbonated } \\
\text { bottled water }\end{array}$ & $\begin{array}{l}\text { VAP frequency, } \\
\text { Duration of ICU } \\
\text { stay, duration of } \\
\text { MV }\end{array}$ & $\begin{array}{l}\text { Number of patients } \\
\text { with findings of } \\
\text { emerging } \\
\text { microorganisms, } \\
\text { positive findings of } \\
\text { bacteria and fungi } \\
\text { species }\end{array}$ \\
\hline
\end{tabular}




\begin{tabular}{|c|c|c|c|c|c|c|c|c|}
\hline $\begin{array}{l}\text { First author year } \\
\text { [ref.] }\end{array}$ & Study design & Male/patients & Age years & Inclusion criteria & Definition of VAP & $\begin{array}{l}\text { Probiotics } \\
\text { intervention }\end{array}$ & Primary outcomes & Secondary outcomes \\
\hline SHIMIZU 2018 [24] & $\begin{array}{l}\text { Single-blind, } \\
\text { randomised, } \\
\text { controlled } \\
\text { trial }\end{array}$ & $47 / 72$ & $\begin{array}{l}\text { Probiotics: } \\
74 \text { (64-82)/ } \\
\text { Control: } \\
\quad 74 \text { (64-81) }\end{array}$ & $\begin{array}{l}\text { Patients }>16 \text { years old } \\
\text { and had been } \\
\text { undergoing MV for } \\
>72 \mathrm{~h} \text { after admission } \\
\text { to the ICU, diagnosed } \\
\text { as sepsis }\end{array}$ & Not mentioned & $\begin{array}{l}\text { Lac+Bif: Yakult BL } \\
\text { Seichoyaku } \\
\left(3 \mathrm{~g} \cdot \text { day }{ }^{-1}\right) \\
\text { contained } 1 \times 10^{8} \\
\text { living bacteria of } \\
\text { the } B \text {. breve } \\
\text { strain Yakult/g } \\
\text { and } 1 \times 10^{8} \text { living } \\
\text { bacteria of the } \\
\text { L. casei strain } \\
\text { Shirota/g and } \\
\text { galactooligo- } \\
\text { saccharides } \\
\left(10 \mathrm{~g} \cdot \text { day }{ }^{-1}\right) \text { were } \\
\text { administered via } \\
\text { nasal tube and } \\
\text { were continued } \\
\text { until oral intake } \\
\text { was initiated }\end{array}$ & $\begin{array}{l}\text { Infectious } \\
\text { complications } \\
\text { including } \\
\text { enteritis, VAP, } \\
\text { and bacteraemia } \\
\text { within } 4 \text { weeks } \\
\text { from admission }\end{array}$ & $\begin{array}{l}\text { Mortality within } \\
4 \text { weeks, faecal } \\
\text { bacterial counts, } \\
\text { organic acid } \\
\text { concentration }\end{array}$ \\
\hline Zeng 2016 [25] & $\begin{array}{l}\text { Prospective, } \\
\text { open-label, } \\
\text { randomised, } \\
\text { controlled } \\
\text { multicentre } \\
\text { trial }\end{array}$ & $138 / 235$ & $\begin{array}{l}\text { Probiotics: } \\
50.2 \pm 18.2 / \\
\text { Control: } \\
54.6 \pm 17.9\end{array}$ & $\begin{array}{l}\text { Patients } \geqslant 18 \text { years with } \\
\text { an expected need of } \\
\text { MV for at least } 48 \mathrm{~h}\end{array}$ & $\begin{array}{l}\text { A new, persistent or } \\
\text { progressive infiltrate on } \\
\text { chest radiographs that } \\
\text { persisted for at least } \\
48 \mathrm{~h} \text { combined with at } \\
\text { least } 2 \text { of the } 3 \text { criteria: } \\
\text { 1) temperature }>38.0^{\circ} \mathrm{C} \\
\text { or }<35.5^{\circ} \mathrm{C} ; 2 \text { l } \\
\mathrm{WBC}>12 \times 10^{3} \text { or } \\
<3 \times 10^{3} \cdot \mathrm{mm}^{-3} \text { and/or } \\
\text { left shift; } 3 \text { l purulent } \\
\text { tracheal aspirates }\end{array}$ & $\begin{array}{l}\text { Bac+Ent: Capsules } \\
\text { containing active } \\
\text { Bacillus subtilis } \\
\text { and Enterococcus } \\
\text { faecalis }\left(4.5 \times 10^{9} /\right. \\
0.25 \mathrm{~g} \text { and } \\
0.5 \times 10^{9} / 0.25 \mathrm{~g}, \\
\text { respectively) } 0.5 \mathrm{~g} \\
\text { three times daily, } \\
\text { for a maximum of } \\
14 \text { days }\end{array}$ & $\begin{array}{l}\text { Microbiologically } \\
\text { confirmed VAP } \\
\text { incidence, the } \\
\text { proportions } \\
\text { eradication of } \\
\text { colonisation, } \\
\text { acquired } \\
\text { colonisation with } \\
\text { PPMOs in the } \\
\text { oropharynx and } \\
\text { stomach }\end{array}$ & $\begin{array}{l}\text { Days on MV, days in } \\
\text { the ICU and in the } \\
\text { hospital after ICU } \\
\text { admission, } \\
\text { mortality lin ICU, in } \\
\text { hospitall, days of } \\
\text { antibiotic use for } \\
\text { VAP, antibiotic-free } \\
\text { days at day } 28 \text {, } \\
\text { carbapenem-free } \\
\text { days at day } 28 \text { and } \\
\text { glycopeptide or } \\
\text { linezolid-free days } \\
\text { at day } 28\end{array}$ \\
\hline
\end{tabular}




\section{TABLE 1 Continued}

\begin{tabular}{|c|c|c|c|c|c|c|c|c|}
\hline $\begin{array}{l}\text { First author year } \\
\text { [ref.] }\end{array}$ & Study design & Male/patients & Age years & Inclusion criteria & Definition of VAP & $\begin{array}{l}\text { Probiotics } \\
\quad \text { intervention }\end{array}$ & Primary outcomes & Secondary outcomes \\
\hline $\begin{array}{l}\text { RoNGRUNGRUANG } 2015 \\
\text { [21] }\end{array}$ & $\begin{array}{l}\text { Prospective, } \\
\text { randomised, } \\
\text { open-label, } \\
\text { controlled } \\
\text { trial }\end{array}$ & $62 / 150$ & $\begin{array}{l}\text { Probiotics: } \\
73 \pm 13.16 \\
\text { (30-94)/ } \\
\text { Control: } \\
69 \pm 18.45 \\
(20-97)\end{array}$ & $\begin{array}{l}\text { Adult patients who were } \\
\text { expected to receive MV } \\
\text { at least } 72 \mathrm{~h} \text { and had } \\
\text { no VAP at enrolment }\end{array}$ & $\begin{array}{l}\text { A new, persistent, or } \\
\text { progressive infiltrate } \\
\text { visible on a chest } \\
\text { radiograph in } \\
\text { combination with at } \\
\text { least } 3 \text { of the } 4 \text { criteria: } \\
\text { 1) temperature }>38^{\circ} \mathrm{C} \\
\text { or }<35.5^{\circ} \mathrm{C} ; 2 \text { ) WBC } \\
>12 \times 10^{3} \text { or } \\
<3 \times 10^{3} \cdot \mathrm{mm}^{-3} \text {; } \\
\text { 3) purulent tracheal } \\
\text { aspirate; } \\
\text { 4) semi-quantitative } \\
\text { culture of tracheal } \\
\text { aspirate samples } \\
\text { positive for pathogenic } \\
\text { bacteria }\end{array}$ & $\begin{array}{l}\text { Lac: } 80 \mathrm{~mL} \text { of } \\
\text { commercially- } \\
\text { available } \\
\text { fermented dairy } \\
\text { product } \\
\text { containing } \\
8 \times 10^{9} \mathrm{CFU} \text { of } \\
\text { Lactobacillus } \\
\text { casei, oral care } \\
\text { after the standard } \\
\text { oral care once } \\
\text { daily, additional } \\
80 \mathrm{~mL} \text { of the } \\
\text { aforementioned } \\
\text { fermented dairy } \\
\text { product was given } \\
\text { once daily for } \\
28 \text { days }\end{array}$ & $\begin{array}{l}\text { Incidence of VAP, } \\
\text { incidence rate of } \\
\text { VAP episodes } \\
\text { per } 1000 \\
\text { ventilator days }\end{array}$ & $\begin{array}{l}\text { Length of hospital } \\
\text { stay, mortality at } \\
\text { day } 28 \text { and } 90, \\
\text { incidence of } \\
\text { diarrhoea, } \\
\text { presence of } \\
\text { resistant bacteria } \\
\text { in oropharyngeal } \\
\text { and rectal swab } \\
\text { samples on day } 0, \\
7 \text { and } 28\end{array}$ \\
\hline BANUPRIYA 2015 [34] & $\begin{array}{l}\text { Open-label, } \\
\text { randomised, } \\
\text { controlled } \\
\text { trial }\end{array}$ & $91 / 150$ & $\begin{array}{l}\text { Probiotics: } \\
2.9 \pm 3.41 / \\
\text { Control: } \\
2.93 \pm 3.77\end{array}$ & $\begin{array}{l}\text { Aged } 12 \text { years or less, } \\
\text { likely to need } M V>48 \mathrm{~h}\end{array}$ & $\begin{array}{l}\text { A new, persisting } \\
\text { radiographical infiltrate } \\
\text { combined with } \\
\text { radiographical evidence } \\
\text { of pulmonary abscess } \\
\text { formation, or } 2 \text { of the } 3 \\
\text { criteria: } 1 \text { ) fever } \\
\text { (increase in the } \\
\text { temperature of at least } \\
1^{\circ} \mathrm{C} \text { and a core } \\
\left.\text { temperature }>38.3^{\circ} \mathrm{C}\right) \text {; } \\
\text { 2) leukocytosis }(25 \% \\
\text { increase in circulating } \\
\text { leukocytes from } \\
\text { baseline or } \\
\left.\text { WBC }>10 \times 10^{3} \cdot \mathrm{mm}^{-3}\right) \text {; } \\
\text { 3) purulent tracheal } \\
\text { aspirate, a positive } \\
\text { blood or pleural fluid } \\
\text { culture with the } \\
\text { microorganisms }\end{array}$ & $\begin{array}{l}\text { Lac+Bif+Str: One } \\
\text { capsule } \\
\text { containing } 2 \\
\text { billion CFU of } \\
\text { Lactobacillus, } 1 \\
\text { billion CFU of } \\
\text { Bifidobacterium, } \\
\text { and } 300 \text { million } \\
\text { CFU of } \\
\text { Streptococcus } \\
\text { thermophilus } \\
\text { were used twice a } \\
\text { day }\end{array}$ & Incidence of VAP & $\begin{array}{l}\text { Duration of hospital } \\
\text { stay, mortality }\end{array}$ \\
\hline
\end{tabular}




\begin{tabular}{|c|c|c|c|c|c|c|c|c|}
\hline $\begin{array}{l}\text { First author year } \\
\text { [ref.] }\end{array}$ & Study design & Male/patients & Age years & Inclusion criteria & Definition of VAP & $\begin{array}{l}\text { Probiotics } \\
\text { intervention }\end{array}$ & Primary outcomes & Secondary outcomes \\
\hline LI 2012 [33] & $\begin{array}{l}\text { Randomised, } \\
\text { control trail }\end{array}$ & $92 / 165$ & $\begin{array}{l}\text { Probiotics: } \\
32.3 \pm 1.5 / \\
\text { Control: } \\
31.6 \pm 1.4 \\
\text { (weeks) }\end{array}$ & $\begin{array}{l}M V \geqslant 48 \mathrm{~h}, \text { no respiratory } \\
\text { tract infection before } \\
\text { orotracheal intubation, } \\
\text { no history of using a } \\
\text { large number of } \\
\text { adrenal cortex } \\
\text { hormone and } \\
\text { immunosuppressive } \\
\text { agents within } 48 \mathrm{~h} \\
\text { before orotracheal } \\
\text { intubation, no immune } \\
\text { deficiency disease }\end{array}$ & $\begin{array}{l}\text { After } 48 \mathrm{~h} \text { of MV, airway } \\
\text { secretion culture was } \\
\text { positive or new } \\
\text { pathogenic bacteria } \\
\text { appeared, new } \\
\text { infiltration shadow } \\
\text { appeared on chest } \\
\text { radiography } \\
\text { accompanied by } \\
\text { increased pulmonary, } \\
\text { clinical fever, WBC } \\
>10.0 \times 10^{9} \cdot \mathrm{L}^{-1}\end{array}$ & $\begin{array}{l}\text { Bif: Probiotics } \\
\text { [Bifidobacterium } \\
\text { triple viable } \\
\text { powder, Shanghai } \\
\text { Xinyi } \\
\text { Pharmaceutical] } \\
\text { at a dose of } \\
0.33 \mathrm{~g} \cdot \text { day }^{-1} \text { after } \\
\text { the start of micro } \\
\text { feeding and } \\
\text { continued until } \\
\text { the end of the } \\
\text { study, if feeding } \\
\text { intolerance } \\
\text { occurs, } \\
\text { immediately fast } \\
\text { and stop oral } \\
\text { probiotics, and } \\
\text { continue oral } \\
\text { probiotics when } \\
\text { milk is reopened, } \\
\text { probiotics fed for } \\
3 \text { days before } \\
\text { blood bacterial } \\
\text { culture }\end{array}$ & $\begin{array}{l}\text { The number of the } \\
\text { bacterial strain } \\
\text { of VAP }\end{array}$ & $\begin{array}{l}\text { The time of bacterial } \\
\text { colonisation, VAP } \\
\text { occurrence }\end{array}$ \\
\hline TAN 2011 [22] & $\begin{array}{l}\text { Prospective, } \\
\text { randomised, } \\
\text { single-blind } \\
\text { study }\end{array}$ & $40 / 52$ & $\begin{array}{l}\text { Probiotics: } \\
\quad 40.5 \pm 13.0 / \\
\text { Control: } \\
\quad 40.8 \pm 12.8\end{array}$ & $\begin{array}{l}\text { Closed head injury alone, } \\
\text { admission within } 24 \mathrm{~h} \\
\text { after trauma, GCS } \\
\text { score between } 5 \text { and } 8, \\
\text { aged } 18 \text { to } 60 \text { years } \\
\text { old, able to be fed via } \\
\text { nasogastric tube within } \\
48 \mathrm{~h} \text { after admission }\end{array}$ & $\begin{array}{l}\text { Pneumonia occurring } \\
\geqslant 48 \mathrm{~h} \text { after } \\
\text { endotracheal } \\
\text { intubation, a new or } \\
\text { progressive } \\
\text { radiographic infiltrate, } \\
\text { at least two clinical } \\
\text { features: } 1 \text { ) } \\
\text { temperature }>38.0^{\circ} \mathrm{C} \text {; } \\
\text { 2) WBC }>12 \times 10^{9} \cdot \mathrm{L}^{-1} \text { or } \\
\text { WBC count }<4 \times 10^{9} \cdot \mathrm{L}^{-1} \text {; } \\
\text { 3) purulent } \\
\text { tracheobronchial } \\
\text { secretions and positive } \\
\text { cultures of } \\
\text { tracheobronchial } \\
\text { secretion }\end{array}$ & $\begin{array}{l}\text { Bif+Lac+Str: Seven } \\
\text { sachets of viable } \\
\text { probiotics leach } \\
\text { sachet containing } \\
0.5 \times 10^{8} \\
\text { Bifidobacterium } \\
\text { longum, } 0.5 \times 10^{7} \\
\text { Lactobacillus } \\
\text { bulgaricus, and } \\
0.5 \times 10^{7} \\
\text { Streptococcus } \\
\text { thermophilus) } \\
\text { three times a day, } \\
\text { which provided a } \\
\text { total of } 109 \\
\text { bacteria }\end{array}$ & $\begin{array}{l}\text { Multiple infections } \\
\text { in the same } \\
\text { patient }\end{array}$ & $\begin{array}{l}\text { The use of antibiotics, } \\
\text { length of ICU stay } \\
\text { and the } 28 \text {-day, } \\
\text { mortality rate }\end{array}$ \\
\hline
\end{tabular}




\begin{tabular}{|c|c|c|c|c|c|c|c|c|}
\hline $\begin{array}{l}\text { First author year } \\
\text { [ref.] }\end{array}$ & Study design & Male/patients & Age years & Inclusion criteria & Definition of VAP & $\begin{array}{l}\text { Probiotics } \\
\text { intervention }\end{array}$ & Primary outcomes & Secondary outcomes \\
\hline MORRow 2010 [23] & $\begin{array}{l}\text { Prospective, } \\
\text { randomised, } \\
\text { double-blind, } \\
\text { placebo- } \\
\text { controlled } \\
\text { trial }\end{array}$ & $86 / 146$ & $\begin{array}{l}\text { Probiotics: } \\
52.5 \pm 19.3 / \\
\text { Control: } \\
54.6 \pm 16.3\end{array}$ & $\begin{array}{l}\text { Adults } \geqslant 19 \text { years old } \\
\text { require } \mathrm{MV} \text { with an } \\
\text { endotracheal tube } \\
\geqslant 72 \mathrm{~h}\end{array}$ & $\begin{array}{l}\text { A new and persistent } \\
\text { infiltrate on chest } \\
\text { radiographs with } 2 \text { of } 3 \\
\text { criteria: } 1 \text { l) temperature } \\
>38.5^{\circ} \mathrm{C} \text { or }<35.0^{\circ} \mathrm{C} ; 2 \text { l } \\
\text { WBC }>10000 \cdot \mathrm{mm}^{-3} \text { or } \\
\left.<3000 \cdot \mathrm{mm}^{-3} ; 3\right) \\
\text { purulent sputum } 4) \\
\text { quantitative BAL } \\
\text { culture with at least } \\
10^{4} \mathrm{CFU} \cdot \mathrm{mL}^{-1} \text { in } \\
\text { patients intubated for } \\
48 \mathrm{~h} \text { or longer }\end{array}$ & $\begin{array}{l}\text { Lac: } 2 \times 10^{9} \mathrm{CFU} \text { of } \\
\text { Lactobacillus } \\
\text { rhamnosus } G G \text { on } \\
\text { a twice-daily } \\
\text { basis, the } \\
\text { contents of one } \\
\text { capsule } \\
\text { containing } \\
10^{9} \mathrm{CFU} \text { of } \\
\text { Lactobacillus were } \\
\text { suspended in } \\
\text { sterile, } \\
\text { water-based } \\
\text { surgical lubricant } \\
\text { and administered } \\
\text { as a slurry to the } \\
\text { oropharynx, the } \\
\text { contents of a } \\
\text { second capsule } \\
\text { containing } 10^{9} \text { of } \\
\text { CFU Lactobacillus } \\
\text { were suspended } \\
\text { in sterile water } \\
\text { and given through } \\
\text { the nasogastric } \\
\text { tube }\end{array}$ & $\begin{array}{l}\text { Microbiologically } \\
\text { confirmed VAP } \\
\text { incidence }\end{array}$ & $\begin{array}{l}\text { Mortality, the time to } \\
\text { occurrence of VAP, } \\
\text { durations of MV, } \\
\text { ICU stay and } \\
\text { hospital stay, } \\
\text { clostridium } \\
\text { difficile-associated } \\
\text { diarrhoea and } \\
\text { another } \\
\text { ICU-associated } \\
\text { diarrhoea, } \\
\text { antibiotic } \\
\text { consumption, and } \\
\text { hospital charges }\end{array}$ \\
\hline BARRAUD 2010 [28] & $\begin{array}{l}\text { Double-blind, } \\
\text { concealed } \\
\text { randomised, } \\
\text { placebo- } \\
\text { controlled } \\
\text { trial }\end{array}$ & $68 / 167$ & $60.7 \pm 15.8$ & $\begin{array}{l}\text { Adult patients under MV } \\
\text { for a predicted period } \\
\text { of at least } 48 \mathrm{~h}\end{array}$ & $\begin{array}{l}\text { A new and persistent } \\
\text { infiltrate on chest } \\
\text { radiograph associated } \\
\text { with at least one of the } \\
\text { following: } 1 \text { ) purulent } \\
\text { tracheal secretions, } \\
\text { temperature } \geqslant 38.3^{\circ} \mathrm{C} \\
\text { and } \mathrm{WBC} \text { count } \\
\geqslant 10 \times 10^{3} \cdot \mu \mathrm{L}^{-1} ; 2 \text { ) } \\
\text { positive quantitative } \\
\text { cultures of distal } \\
\text { pulmonary secretions } \\
\text { obtained from BAL } \\
\text { (significant threshold } \\
\text { more than } \\
10^{4} \mathrm{CFU} \cdot \mathrm{mL}^{-1} \text { ) }\end{array}$ & $\begin{array}{l}\text { Lac+Bif: } 2 \times 10^{10} \text { of } \\
\text { revivable bacteria } \\
\text { (mainly } \\
\text { Lactobacillus } \\
\text { rhamnosus } G G, \\
\text { but also } \\
\text { Lactobacillus } \\
\text { casei, } \\
\text { Lactobacillus } \\
\text { acidophilus, and } \\
\text { Bifidobacterium } \\
\text { bifidum) once a } \\
\text { day until } \\
\text { successful } \\
\text { weaning }\end{array}$ & 28-day mortality & $\begin{array}{l}\text { Infection and } \\
\text { diarrhoea, length } \\
\text { of stay in ICU of } \\
\text { hospital, resolution } \\
\text { of organ failure at } \\
28 \text { days }\end{array}$ \\
\hline
\end{tabular}




\begin{tabular}{|c|c|c|c|c|c|c|c|c|}
\hline $\begin{array}{l}\text { First author year } \\
\text { [ref.] }\end{array}$ & Study design & Male/patients & Age years & Inclusion criteria & Definition of VAP & $\begin{array}{l}\text { Probiotics } \\
\text { intervention }\end{array}$ & Primary outcomes & Secondary outcomes \\
\hline KNIGHT 2009 [27] & $\begin{array}{l}\text { Prospective, } \\
\text { randomised, } \\
\text { double-blind, } \\
\text { placebo- } \\
\text { controlled } \\
\text { trial }\end{array}$ & $161 / 259$ & $\begin{array}{l}\text { Probiotics: } \\
\text { 49.5 } \pm 19.6 / \\
\text { Control: } \\
50.0 \pm 18.5\end{array}$ & $\begin{array}{l}\text { Expected to require MV } \\
\text { for at least } 48 \mathrm{~h} \text {, no } \\
\text { contraindications to } \\
\text { enteral nutrition }\end{array}$ & $\begin{array}{l}\text { A new progressive, or } \\
\text { persistent infiltration } \\
\text { on chest radiograph } \\
\text { plus at least two of the } \\
\text { following: 1) } \\
\text { temperature }>38.0^{\circ} \mathrm{C} \text {; } \\
\text { 2) } \mathrm{WBC}>12 \times 10^{3} \cdot \mu \mathrm{L}^{-1} \text { or } \\
<4 \times 10^{3} \cdot \mu \mathrm{L}^{-1} ; 3 \text { ) } \\
\text { purulent } \\
\text { tracheobronchial } \\
\text { secretions }\end{array}$ & $\begin{array}{l}\text { Synbiotic } 2000 \\
\text { FORTE: At least } \\
2 \text { days of either } \\
\text { Synbiotic } 2000 \\
\text { FORTE twice a } \\
\text { day, or a } \\
\text { crystalline } \\
\text { cellulose based } \\
\text { placebo, Synbiotic } \\
\text { 2000 FORTE } \\
\text { contains } \\
\text { Pediococcus } \\
\text { pentosaceus, } \\
\text { Leuconostoc } \\
\text { mesenteroides, } \\
\text { Lactobacillus } \\
\text { paracasei subsp. } \\
\text { paracasei and } \\
\text { Lactobacillus } \\
\text { plantarum as } \\
\text { probiotics }\end{array}$ & Incidence of VAP & $\begin{array}{l}\text { Oropharyngeal flora, } \\
\text { duration of MV and } \\
\text { VAP rates per } 1000 \\
\text { ventilator days, } \\
\text { length of ICU stay, } \\
\text { mortality in ICU } \\
\text { and hospital }\end{array}$ \\
\hline $\begin{array}{l}\text { Giamarellos- } \\
\text { Bourboulis } 2009 \\
\text { [26] }\end{array}$ & $\begin{array}{l}\text { Double-blind, } \\
\text { placebo- } \\
\text { controlled, } \\
\text { multicentre, } \\
\text { randomised } \\
\text { clinical trial }\end{array}$ & $? / 72$ & $\begin{array}{l}\text { Probiotics: } \\
52.9 / \\
\text { Control: } \\
55.9\end{array}$ & $\begin{array}{l}\text { Patients with severe } \\
\text { multiple organ injuries } \\
\text { necessitating } \\
\text { emergency tracheal } \\
\text { intubation and } \\
\text { ventilation support and } \\
\text { subsequent } \\
\text { hospitalisation in ICU }\end{array}$ & $\begin{array}{l}\text { Patients presenting with } \\
\text { all of the following: } \\
\text { 1) new or persistent } \\
\text { consolidation in lung } \\
\text { radiograph; 2) purulent } \\
\text { TBS; 3) clinical } \\
\text { pulmonary infection } \\
\text { score (CPIS)>6 }\end{array}$ & $\begin{array}{l}\text { Synbiotic } 2000 \\
\text { FORTE: The } \\
\text { formula Synbiotic } \\
2000 F O R T E \text { was } \\
\text { diluted in } 100 \mathrm{~mL} \\
\text { of tap water and } \\
\text { administered by a } \\
\text { nasogastric tube } \\
\text { or through } \\
\text { gastrostomy once } \\
\text { daily for } 15 \\
\text { consecutive days } \\
\text { after admission }\end{array}$ & Incidence of VAP & $\begin{array}{l}\text { Incidence of } \\
\text { bloodstream } \\
\text { infections, } \\
\text { incidence and } \\
\text { comparative time } \\
\text { of primary } \\
\text { bacteraemia, } \\
\text { comparative serum } \\
\text { levels of WBCs and } \\
\text { CRP of patients } \\
\text { with primary } \\
\text { bacteraemia and } \\
\text { with VAP }\end{array}$ \\
\hline
\end{tabular}




\begin{tabular}{|c|c|c|c|c|c|c|c|c|}
\hline $\begin{array}{l}\text { First author year } \\
\text { [ref.] }\end{array}$ & Study design & Male/patients & Age years & Inclusion criteria & Definition of VAP & $\begin{array}{l}\text { Probiotics } \\
\text { intervention }\end{array}$ & Primary outcomes & Secondary outcomes \\
\hline FoRESTIER 2008 [29] & $\begin{array}{l}\text { Prospective, } \\
\text { randomised, } \\
\text { double-blind, } \\
\text { placebo- } \\
\text { controlled } \\
\text { pilot study }\end{array}$ & $146 / 208$ & $\begin{array}{l}\text { Probiotics: } \\
60(18-91) / \\
\text { Control: } \\
57(18-80)\end{array}$ & $\begin{array}{l}\text { Patients } \geqslant 18 \text { years with a } \\
\text { stay longer than } 48 \mathrm{~h} \\
\text { and a nasogastric } \\
\text { feeding tube }\end{array}$ & $\begin{array}{l}\text { According mostly to } \\
\text { CDC's NHSN criteria: } \\
\text { 1) at least one positive } \\
\text { sample } \\
\text { (bronchoalveolar } \\
\text { mini-lavage } \\
>10^{3} \mathrm{CFUs} \cdot \mathrm{mL}^{-1} \text { or } \\
\text { endotracheal aspirate } \\
\text { with }>10^{5} \mathrm{CFUs} \cdot \mathrm{mL}^{-1} \text { ) } \\
\text { 2) the presence of one } \\
\text { or several new } \\
\text { abnormal } \\
\text { radiographical and } \\
\text { progressive } \\
\text { parenchymatous } \\
\text { infiltrates; 3) one of the } \\
\text { following signs: } \\
\text { purulent sputum } \\
\text { production, fever } \\
\text { (temperature }>38.5^{\circ} \mathrm{C} \text { ), } \\
\text { pathogenic bacteria in } \\
\text { blood culture without } \\
\text { other infection source, } \\
\text { and BAL }>5 \% \text { cells with } \\
\text { intracellular bacteria }\end{array}$ & $\begin{array}{l}\text { Lac: } L \text {. casei } \\
\text { rhamnosus } \\
\left(10^{9} \mathrm{CFU}\right), \text { twice } \\
\text { daily }\end{array}$ & $\begin{array}{c}\text { The time of first } \\
P \text {. aeruginosa } \\
\text { acquisition }\end{array}$ & $\begin{array}{l}\text { Whether respiratory } \\
\text { tract infection or } \\
\text { colonisation due to } \\
P \text {. aeruginosa, to } \\
\text { evaluate the ability } \\
\text { of } L \text {. casei } \\
\text { rhamnosus to } \\
\text { persist in the } \\
\text { stomach }\end{array}$ \\
\hline KLARIN 2008 [9] & $\begin{array}{l}\text { Randomised, } \\
\text { controlled, } \\
\text { open pilot } \\
\text { study }\end{array}$ & $22 / 44$ & $\begin{array}{l}\text { Probiotics: } \\
70 \text { (20-87)/ } \\
\text { Control: } \\
70 \text { (43-81) }\end{array}$ & $\begin{array}{l}\text { Patients } \geqslant 18 \text { years, } \\
\text { critically ill with an } \\
\text { anticipated need for } \\
\text { MV of at least } 24 \mathrm{~h} \text {, not } \\
\text { moribund, not } \\
\text { suffering from } \\
\text { pneumonia at } \\
\text { admission, no } \\
\text { fractures in the facial } \\
\text { skeleton or the base of } \\
\text { the skull, no oral } \\
\text { ulcers, not immune } \\
\text { deficient, not a carrier } \\
\text { of HIV or viral hepatitis }\end{array}$ & $\begin{array}{l}\text { A new, persistent or } \\
\text { progressive infiltrate on } \\
\text { chest radiograph } \\
\text { combined with at least } \\
3 \text { of the other } 4 \text { criteria: } \\
\text { 1) a purulent tracheal } \\
\text { aspirate; } 2 \text { ) positive } \\
\text { culture of tracheal } \\
\text { aspirates occurring } \\
\text { after } 48 \mathrm{~h} \text { of } \mathrm{MV} ; 3 \text { ) } \\
\text { rectal or urine bladder } \\
\text { temperature }>38.0^{\circ} \mathrm{C} \text { or } \\
<35.5^{\circ} \mathrm{C} ; 4 \text { ) WBC } \\
>12 \times 10^{9} \cdot \mathrm{L}^{-1} \text { or } \\
<3 \times 10^{9} \cdot \mathrm{L}^{-1}\end{array}$ & $\begin{array}{l}\text { Lac: Lp299 } \\
\text { (Lactobacillus } \\
\text { plantarum 299) } \\
\text { twice a day, } \\
\text { subsequent } \\
\text { cleansing was } \\
\text { performed with } \\
\text { gauze swabs } \\
\text { soaked in } \\
\text { carbonated } \\
\text { bottled water }\end{array}$ & \multicolumn{2}{|c|}{$\begin{array}{l}\text { Pathogenic bacteria analysis in oropharynx } \\
\text { and trachea }\end{array}$} \\
\hline
\end{tabular}




\begin{tabular}{|c|c|c|c|c|c|c|c|}
\hline $\begin{array}{l}\text { First author year } \\
\text { [ref.] }\end{array}$ & Study design & Male/patients & Age years & Inclusion criteria & Definition of VAP & $\begin{array}{l}\text { Probiotics } \\
\text { intervention }\end{array}$ & Primary outcomes Secondary outcomes \\
\hline $\begin{array}{l}\text { SPINDLER-VESEL } 2007 \\
\text { [31] }\end{array}$ & $\begin{array}{l}\text { Prospective, } \\
\text { randomised, } \\
\text { double-blind } \\
\text { study }\end{array}$ & $88 / 113$ & $41 \pm 18.9$ & $\begin{array}{l}\text { Multiple injured patients } \\
\text { with an ISS of }>18 \text { and } \\
\text { at least a 4-day ICU } \\
\text { stay }\end{array}$ & Not mentioned & \begin{tabular}{l} 
Synbiotic 2000 \\
FORTE: The \\
contents of the \\
sachets 110 \\
\multicolumn{10}{c}{10} \\
Pediococcus \\
pentosaceus \\
$5-33: 3,10^{10}$ \\
Lactococcus \\
raffinolactis \\
$32-77: 1,10^{10}$ \\
Lactobacillus \\
paracasei subsp. \\
paracasei $19,10^{10}$ \\
Lactobacillus \\
plantarum 2362 \\
and the fibres) \\
were dissolved in \\
$100 \mathrm{~mL}$ of \\
lukewarm sterile \\
water
\end{tabular} & $\begin{array}{l}\text { Incidence of infection (such as VAP), } \\
\text { duration of MV, multiple organ failure } \\
\text { scores, length of ICU stay }\end{array}$ \\
\hline
\end{tabular}

VAP: ventilation-associated pneumonia; MV: mechanical ventilation; BAL: bronchoalveolar lavage; Lac: Lactobacillus (casei, plantarum, rhamnosus, bulgaricus; acidophilus); Bif: Bifidobacterium (breve, longum, bifidum); Str: Streptococcus thermophilus; ICU: intensive care unit; WBC: white blood cell; Bac: Bacillus subtilis; Ent: Enterococcus (faecalis); PPMO: potentially pathogenic microorganism; CFU: colony-forming unit; GCS: Glasgow coma score; TBS: tracheobronchial secretions; CPIS: clinical pulmonary infection score; CRP: C-reactive protein; CDC: the US Centers for Disease Control and Prevention; NHSN: National Healthcare Safety Network; ISS: injury severity score. Ages are presented as median (range) or mean \pm SD. 
FIGURE 2 The risk of bias summary of 15 randomised control trials based on bias assessment of selection, allocation concealment, performance, detection, attrition and publication.

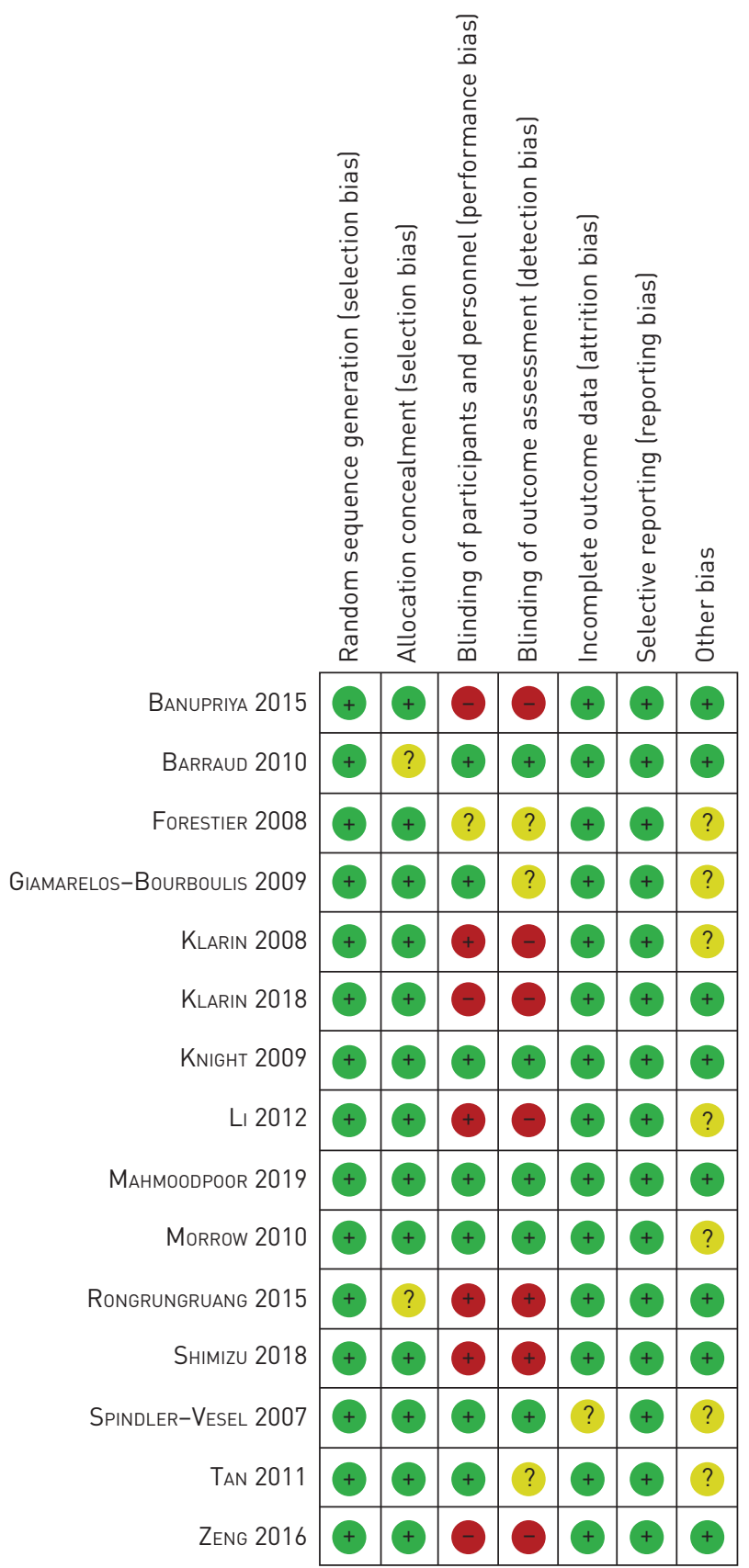

quantitative microbiological confirmed VAP (SMD, $-0.19 ; 95 \% \mathrm{Cl},-0.49$ to $0.11 ; \mathrm{p}=0.22 ; \mathrm{I}^{2}=56 \%$ ) both indicated no significant reduction of duration of $\mathrm{MV}$ with high heterogeneity. However, the nonquantitative microbiological confirmed VAP subgroup suggested probiotics significantly reduced the duration of $\mathrm{MV}(\mathrm{SMD},-0.61 ; 95 \% \mathrm{Cl},-0.95$ to $-0.27 ; \mathrm{p}=0.004)$. Only three studies reported a significantly statistical difference in the duration of $\operatorname{MV}[9,32,34]$. In addition, KLARIN et al. [9, 30] detected a significant difference $(\mathrm{p}<0.0001)$ in 2008 but reported opposite conclusions after analysing a larger sample size as part of a multicentre collaboration in 2018. Generally, duration of MV is a variable influenced by multiple factors, such as diverse complications, the basic physic status, age, and the reasons for ICU admission of the patients. Patients with thoracic trauma and respiratory failure are likely to have a long length of MV, which likely accounts for the high heterogeneity. For instance, OudHuIs et al. [35] reported longer length of MV (mean difference (MD) \pm SD, 16.7 \pm 23.6 ) in patients with higher APACHE score $(\mathrm{MD} \pm \mathrm{SD}, 23 \pm 7.7)$, mostly diagnosed as respiratory insufficient and sepsis shock, than the study by KNIGHT et al. [27] ( $\mathrm{MD} \pm \mathrm{SD}, 5 \pm 5.19)$, in which patients were mostly assessed after surgery (with a median APACHE score of 17; range, 12 to 23) [27]. Sensitivity analysis by removing each trial suggested the same 


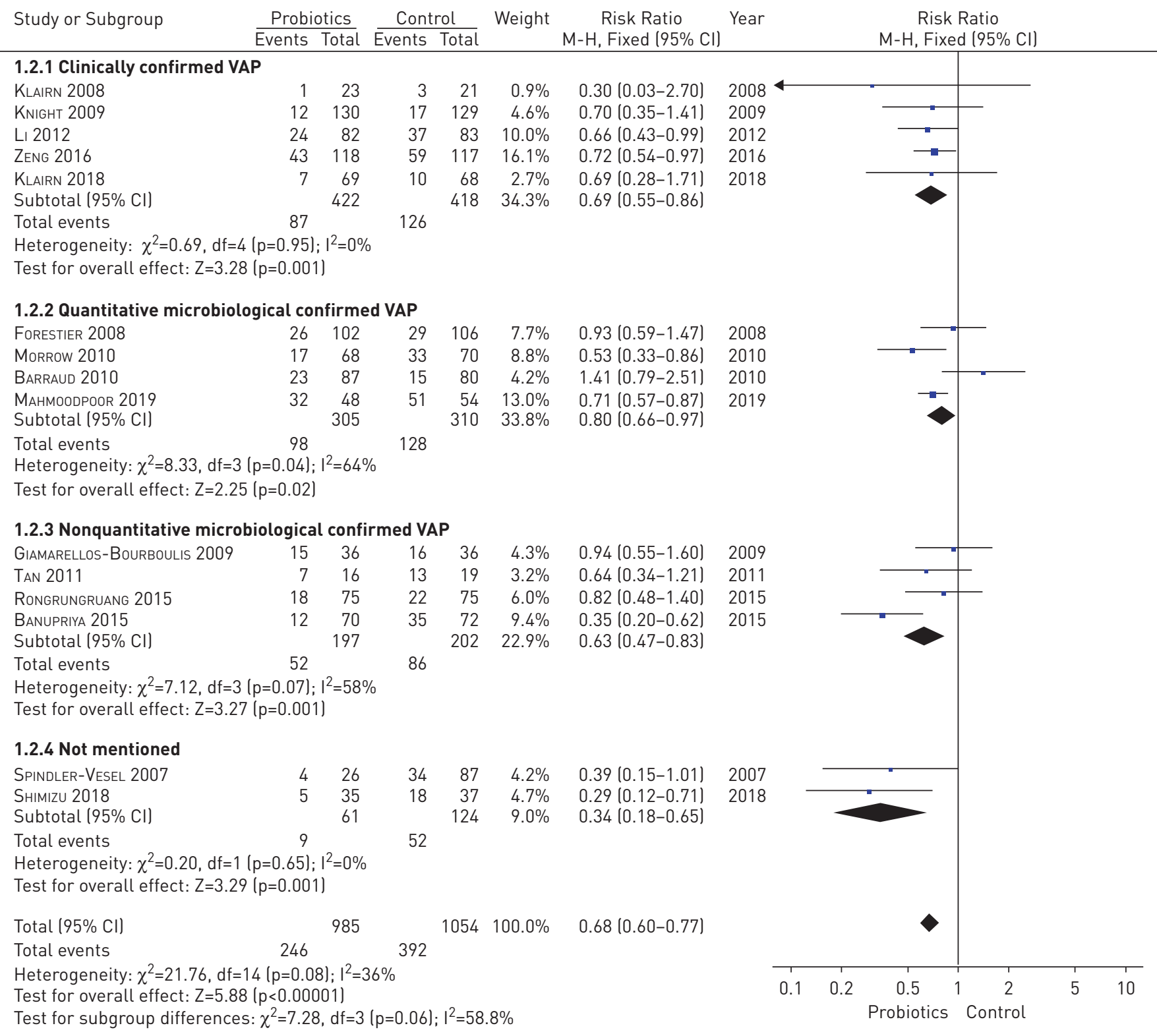

FIGURE 3 The pooled and subgroup analysis for the effect of probiotics on morbidity of ventilator-associated pneumonia based on 15 studies, involving 2039 patients compared with the placebo group.

results as in the overall analysis. The funnel plot analysis was unsymmetrical, suggesting that a publication bias exists (supplemental figure 3).

No change of the length of ICU stay in probiotics treated patients

Eleven studies assessed length of ICU stay, and the pooled meta-analysis with random-effects model indicated no significant reduction of ICU stay in the probiotics group compared to the control group $(\mathrm{SMD},-0.20,95 \% \mathrm{Cl},-0.46$ to $0.06 ; \mathrm{p}=0.13)[9,21-25,27,28,31,32,34]$. However, the results were limited due to the high heterogeneity $\left(\mathrm{p}<0.00001, \mathrm{I}^{2}=83 \%\right)$ (supplemental figure 4$)$. We conducted a subgroup analysis and found that no significant reduction of ICU stay in the probiotics group compared to control group for patients diagnosed by clinical (SMD, $-0.12,95 \% \mathrm{Cl},-0.29$ to $0.05 ; \mathrm{p}=0.17 ; \mathrm{I}^{2}=0 \%$ ) or quantitative microbiological criteria (SMD, $-0.39,95 \% \mathrm{Cl},-1.03$ to $0.26 ; \mathrm{p}=0.24 ; \mathrm{I}^{2}=90 \%$ ) and nonquantitative microbiological criteria ( $\mathrm{SMD},-0.19,95 \% \mathrm{Cl},-1.12$ to $0.74 ; \mathrm{p}=0.68 ; \mathrm{I}^{2}=94 \%$ ). A previous study indicated that patients with positive microbiology had more frequently decreased consciousness as the cause of ICU admission and longer ICU stays before the diagnosis of pneumonia [40]. Patients with different aetiology have various rehabilitation abilities, which impact the duration of ICU stay. The findings of MAHMOODPOOR et al. [32], BANUPRIYA et al. [34], and TAN et al. [22] are inconsistent with our 
analysis, and we found that their subjects were gathered from a specific population, such as adults with surgical reasons of neurological manifestations ( $\mathrm{SMD},-1.13 ; 95 \% \mathrm{Cl},-1.15$ to $-0.71 ; \mathrm{p}=0.007$ ), those with severe traumatic brain injury $(\mathrm{SMD},-0.66 ; 95 \% \mathrm{Cl},-1.22$ to $-0.10 ; \mathrm{p}=0.034)$, and children with neurological problems (SMD, $-0.62 ; 95 \% \mathrm{Cl},-0.96$ to $-0.28 ; \mathrm{p}<0.001$ ). The remaining eight studies all found no significant difference in duration of ICU stay $[9,21,23-25,27,28,31]$. Rongrungruang et al. [21], who assessed the data of probiotics effect on patients with basic oral application of CHX and found that the length of ICU stay in the probiotics group was longer than in the control group (MD, 30.5 versus 19 ), although without reaching statistically significance (SMD, $0.67 ; 95 \% \mathrm{Cl}, 0.34$ to $0.99 ; \mathrm{p}=0.46$ ). Sensitivity analysis by removing one trail suggested an obvious change of ICU stay length after prebiotics treatment (SMD, $-0.29,95 \% \mathrm{Cl},-0.51$ to $-0.08 ; \mathrm{p}=0.007$ ). After removing the data of RongruNGRUANG et al. [21], the nonquantitative microbiologically confirmed VAP subgroup also suggested a significant reduction of ICU duration after probiotic treatment $(\mathrm{SMD},-0.63,95 \% \mathrm{Cl},-0.92$ to $-0.34 ; \mathrm{p}<0.0001$, $\mathrm{I}^{2}=0 \%$ ). The subjects of the trial of Rongrungruang et al. [21] were older, female, and had diverse severe comorbidities, of whom the length of the ICU stay was not just associated with VAP but influenced by complex individual status. Therefore, this trial's result was not of sufficiently high quality to evaluate the effect of probiotics on duration of ICU stay. No obvious publication bias was observed by funnel plot analysis (supplemental figure 5).

\section{Mortality analysis of critically ill patients}

Eleven studies assessed the mortality of patients and the meta-analysis suggested that there was no significant difference in total mortality in the probiotic group (145 of 716, 20.25\%) versus the control group $(160$ of $684,23.39 \%)$ in the fixed-effects model (supplemental figure 6) $[9,21-28,32,34]$. RONGRUNGRUANG et al. [21] and BARRAUD et al. [28] recorded the 28-day mortality and 90-day mortality of the participations, whereas TAN et al. [22] only evaluated the 28-day mortality of their patients. As shown in supplemental figure 6, the eight remaining trials and that of BARRAUD et al. [28] all evaluated overall mortality $[9,23-27,32,34]$. Due to such a distinction in recorded statistics, a subgroup analysis was carried out to achieve a more convincible conclusion. Our meta-analysis result was that there was no significant difference in 28-day mortality between probiotic (43 of 188, 22.9\%) and control (41 of 181 , $22.7 \%$ ) groups (risk ratio, 1.01; $95 \% \mathrm{Cl}, 0.69$ to $1.47 ; \mathrm{p}=0.97$ ), 90-day mortality in the probiotic (52 of 162 , $32 \%$ ) and control (50 of 155, 32.3\%) groups (risk ratio, 1.00; $95 \% \mathrm{Cl}, 0.72$ to $1.37 ; \mathrm{p}=0.99$ ), and overall mortality in the probiotic (111 of $615,18 \%)$ versus control (126 of 583, 21.6\%) groups (risk ratio, 0.84 ; $95 \% \mathrm{Cl}, 0.67$ to $1.05 ; \mathrm{p}=0.13$ ). At the same time, heterogeneity was not observed in 28-day mortality $\left(\mathrm{I}^{2}=0 \%, \mathrm{p}=0.72\right), 90$-day mortality $\left(\mathrm{I}^{2}=0 \%, \mathrm{p}=0.82\right)$, and overall mortality $\left(\mathrm{I}^{2}=0 \%, \mathrm{p}=0.56\right)$. All of the above meta-analysis results suggested that prophylactic use of probiotics was not beneficial in decreasing critically ill patients' mortality. Interestingly, BARRAUD et al. [28] performed a subgroup analysis based on different severities of sepsis and observed a significant mortality reduction among these severe septic patients treated with probiotics (odds ratio, $0.38 ; 95 \% \mathrm{CI}, 0.16$ to $0.93 ; \mathrm{p}=0.035$ ). By contrast, nonsevere septic patients administered by probiotics had higher mortality at 28 and 90 days than placebo-treated patients, which could be confirmed by a survival analysis: the hazard ratio for 90 -day death was 3.40 ( $95 \%$ $\mathrm{Cl}, 1.18$ to $7.64 ; \mathrm{p}=0.02$ ). This result, opposite to previous studies that deleterious effects of probiotics are only expected to appear among the more severe patients, makes the population and time point of probiotic regimen controversial [41]. Besides, fatal adverse effects were not observed among all studies. Funnel plot analysis indicated no obvious publication bias regarding the total mortality (supplemental figure 7).

\section{Discussion}

Many studies have suggested probiotics' preventive effect, among which Giamarellos-Bourboulis et al. [26] reported the prophylactic effect for VAP of Acinetobacter baumannii [26], and Forestier et al. [29] reported that probiotics delayed respiratory tract infection by P. aeruginosa. However, KNiGHT et al. [27], BARRAud et al. [28], and OudHuis et al. [35] found no evidence that probiotics help to prevent VAP. As MAHMOODPoor et al. [32] have described, their result was inconclusive due to many influencing factors, such as different definitions of VAP occurrence, the sample size, the number of centres, degree of compliance to VAP prevention bundles, and types of probiotic species. A recent network meta-analysis conducted by FAN et al. [42] suggested that "Bifidobacterium longum+Lactobacillus bulgaricus+Streptococcus thermophiles" have higher efficacy in decreasing the incidence of VAP than other probiotic regimens. As illustrated in table 1, the probiotics regimens of the included studies varied, which might be a potential source of the heterogeneity and deserving attention. This systemic review comprehensively analysed the data of ICU patients from 15 RCTs to assess the preventive effect of probiotics for VAP. We found that the administration of probiotics played a significant role in reducing VAP incidence, but did not lead to a statistically significant reduction in length of ICU stay, duration of MV, mortality short- (28-day) or long-term (90-day), or overall mortality. Serious adverse effects or fatal complications were not observed among the studies. Recently, two larger multicentre RCTs have been registered and the recruitment as well 
as analysis are ongoing for now [43,44], which will further enrich our understanding. So why is it that probiotics still cannot reduce mortality, despite significantly preventing VAP? The results of clinical trials and animal experiments both supported the benefits of probiotics in immunomodulation and fighting against pathogens. In our opinion, the direct effect of probiotics is to maintain the balance of microorganism. Additionally, probiotics might improve patients' overall status by systemic immunomodulation, an indirect method that influenced by individual immune system. Critically ill patients always have severe primary aetiology and multiple comorbidities, and the recovery of the most severe symptom determined the duration of MV, duration of ICU stay and mortality. Besides, the different efficacy of the dissimilar probiotic regimens should also be taken consideration. Synbiotic 2000FORTE was suggested to have potential effect in reducing mortality according to a network meta-analysis of 14 RCTs [42].

Among the studies included in our meta-analysis, only two RCTs applied the Synbiotic 2000FORTE. This might be an explanation why probiotics can reduce incidence of VAP but cannot decrease mortality.

The protective role of gut microbiota in host protection against bacterial pneumonia has been demonstrated using animal models. Schuis et al. [45] found increased bacterial dissemination, inflammation, organ failure, and increased mortality following infection with Streptococcus pneumoniae in a mouse gut-bacteria-depleted model. These authors also observed that faecal microbiota transplantation to gut microbiota-depleted mice resulted in decreased bacterial counts and tumour necrosis factor- $\alpha$ and interleukin (IL)-10 levels in the lung early after pneumococcal infection compared to the gut-bacteria-depleted group. Using a Staphylococcus aureus pneumonia model, GAUGUET et al. [46] found that mice in their segmented filamentous bacteria (SFB)-deficit group had a higher bacterial burden in the lungs, lung inflammation, and mortality than mice colonised with SFB. VIEIRA et al. [47] reported that live probiotics release metabolites (e.g. acetate) that modulate the inflammatory response against infection in a mice model of Klebsiella pneumoniae, and that these effects involve reactive oxygen species generation by alveolar macrophages, IL-10 generation, interference with nuclear factor- $\kappa \mathrm{B}$ pathway, and transduction of the Mal/TIRAP signal pathway. KHAILOva et al. [48] observed reduced bacterial counts, reduced histological lung injury, lower number of infiltrating neutrophils, and decreased mice mortality in a Pseudomonas pneumonia model after administration of Lactobacillus rhamnosus GG (LGG), which appeared to be mediated by immunomodulatory cells and molecules.

Although the mechanisms by which probiotics produce a preventive effect are still poorly understood, multiple valuable scientific advances have been published $[49,50]$. Several studies have reported that probiotics play a positive role in fighting against pathogens via immunomodulatory activities. Probiotics can facilitate protection against influenza virus infection by enhancing mucosal secretory IgA production and activating humoral and cellular immune response [51-53]. When comparing a gut-microbe-depleted group and control group, there was a difference in the super pathway of cholesterol biosynthesis and zymosterol biosynthesis canonical signal pathways, which are associated with antibacterial effector functions of alveolar macrophages [45]. The gut microbiota enhances the response of alveolar macrophages to bacterial virulence factors and phagocytosis capacity. The administration of LGG can increase gene expression of Foxp3, and the Foxp3 transcription factor controls the expression of CD4 and CD25, the maker of regulatory $\mathrm{T}\left(\mathrm{T}_{\text {reg }}\right)$ cells. Traditionally, $\mathrm{T}_{\text {reg }}$ cells can regulate inflammation directly or through releasing anti-inflammatory cytokines, such as IL-10 [48]. However, the protective effect of Lactobacillus is demonstrated as not only irrelevant with IL-10 in respiratory virus infection but is also independent of specific interaction with pattern recognition receptors, Toll-like receptor (TLR2) and NOD2 in vitro, which suggests that more possible mechanisms needed to be explored [53]. Type 17 helper $\mathrm{T}\left(\mathrm{T}_{\mathrm{H}} 17\right)$ cell innate immune response is also activated by probiotics as bronchoalveolar lavage fluid levels of IL-22 (one of the main $\mathrm{T}_{\mathrm{H}} 17$ effector cytokines) were significantly increased in a mouse model, subsequently contributing to neutrophil attraction and production of antimicrobial peptides during pulmonary S. aureus infection [46]. Interestingly, the intestinal microbiota, including SFB, can regulate pulmonary adaptive immune responses during acute fungal infection in the lung $[54,55]$. In summary, all findings described above prove the immunomodulatory properties of the antimicrobial activity of probiotics and indicate probiotics might play a positive role in treating pneumonia. Studies about animals and cells fail to show systemic conclusions of comprehensive spectrum pathogens of pneumonia so far. Therefore, further analyses are required. Nevertheless, because of heterogeneity of age, type and degree of disease, treatment duration, dose, type of strains, and outcome measures, the effect of probiotics in clinical trials involving ICU patients remains unclear. A trial performed on patients with predicted severe acute pancreatitis by BESSELINK et al. [56] suggested that probiotic prophylaxis did not reduce the risk of infectious complications and was associated with an increased risk of mortality. BARRAUD et al. [28] prematurely stopped their trial due to the risk reported by BEsselink et al. [56], which might impact the reliability of the study. None of the included studies reported fatal side effects of administration of probiotics, which might be a result of researchers avoiding selecting this category of ICU patients. 
Probiotics showed a preventive effect on severe septic patients but were associated with a high risk of death in nonsevere septic patients, as BARRAUD et al. [28] observed. These findings might be explained by the fact that septic patients were sicker than nonseptic patients and that a treatment effect might have been only apparent in the more severe patients. Age is likely a factor that needs to be considered when interpreting these results because two studies were conducted on children $[33,34]$, to whom probiotics have a different effect compared to adults. It is well-known that children's microflora is dynamic and begins to resemble that of adults at around 2 years of age [34]. Besides, diverse controlled conditions, such as definitions of VAP, the sample size, the number of centres enrolled in the study, compliance to the prevention bundles, different species, or combinations of bacteria lead to the heterogeneity among the results. The quality and quantity of randomised evidence remain insufficient to draw firm conclusion about the clinical effects of probiotics, neither supporting nor discouraging their systematic administration in critically ill patients.

Our meta-analysis review has several limitations: 1) among the included studies, only seven were double-blind trials, and the remainder were single-blind or open-label trials; 2) two studies were performed on children, whereas the remaining studies were all carried out on adults; 3 ) the heterogeneity derived from age, race, baseline treatment, dose, and type of strains possibly affected the precision of our conclusions; 4) possible publication bias should not be ignored as we failed to identify articles showing neutral or negative outcomes and unpublished studies; 5) we cannot exclude the possibility that our conclusions are erroneous because some critical information was ignored; and 6) the diverse diagnosis criteria of VAP and admission criteria of the ICU might have contributed to inconsistency. Though there are some limitations to this review, we attempted to eradicate selection bias by selecting the articles that were strictly subjected to inclusion and exclusion criteria. Also, we performed this study by strictly following the Cochrane Handbook for Systematic Reviews of Interventions 5.1 guidelines, which should minimise bias as much as possible.

\section{Conclusion}

Our systematic review and meta-analysis demonstrated that probiotics played a positive role in preventing VAP without impacting duration of MV, length of ICU stay, or mortality. Well-designed multicentre trials are also needed to further establish the efficacy of probiotics in prevention of VAP.

Author contributions: Bao-ping Tian contributed to the conception and design of this study. Article selection was performed by Jie Zhao and Lei-qing Li. The data from each eligible study were extracted by Cheng-yang Chen and Gen-sheng Zhang. Bao-ping Tian and Wei Cui performed the data analysis. Any dissenting opinion was resolved by a third investigator. All authors reviewed the draft and approved the submission.

Conflict of interest: None declared.

Support statement: This work was supported by the National Natural Science Foundation of China (81700023), the Zhejiang Provincial Natural Science Foundation of China (LY20H010002), and the Medical and Health Research Program of Zhejiang Province (2019RC181) to Bao-ping Tian. Funding information for this article has been deposited with the Crossref Funder Registry.

\section{References}

1 Metersky ML, Kalil AC. Management of ventilator-associated pneumonia: guidelines. Clin Chest Med 2018; 39: 797-808.

2 Schreiber MP, Shorr AF. Challenges and opportunities in the treatment of ventilator-associated pneumonia. Expert Rev Anti Infect 2017; 15: 23-32.

3 Zhang Y, Yao Z, Zhan S, et al. Disease burden of intensive care unit-acquired pneumonia in China: a systematic review and meta-analysis. Int J Infect Dis 2014; 29: 84-90.

4 Melsen WG, Rovers MM, Groenwold RH, et al. Attributable mortality of ventilator-associated pneumonia: a meta-analysis of individual patient data from randomised prevention studies. Lancet Infect Dis 2013; 13: 665-671.

5 Muscedere J, Sinuff T, Heyland DK, et al. The clinical impact and preventability of ventilator-associated conditions in critically ill patients who are mechanically ventilated. Chest 2013; 144: 1453-1460.

6 Kollef MH, Hamilton CW, Ernst FR. Economic impact of ventilator-associated pneumonia in a large matched cohort. Infect Control Hosp Epidemiol 2012; 33: 250-256.

7 Vincent JL, de Souza Barros D, Cianferoni S. Diagnosis, management and prevention of ventilator-associated pneumonia: an update. Drugs 2010; 70: 1927-1944.

8 Motos A, Yang H, Yang M, et al. Perspectives on synthetic pharmacotherapy for the treatment of nosocomial pneumonia. Expert Opin Pharmacother 2019; 20: 1439-1448.

9 Klarin B, Molin G, Jeppsson B, et al. Use of the probiotic Lactobacillus plantarum 299 to reduce pathogenic bacteria in the oropharynx of intubated patients: a randomised controlled open pilot study. Crit Care 2008; 12: R136.

10 Loo CY, Lee WH, Young PM, et al. Implications and emerging control strategies for ventilator-associated infections. Expert Rev Anti Infect Ther 2015; 13: 379-393.

11 Ghosh S, van Heel D, Playford RJ. Probiotics in inflammatory bowel disease: is it all gut flora modulation? Gut 2004; 53: 620-622. 
12 Siempos II, Ntaidou TK, Falagas ME. Impact of the administration of probiotics on the incidence of ventilator-associated pneumonia: a meta-analysis of randomized controlled trials. Crit Care Med 2010; 38: 954-962.

13 Bo L, Li J, Tao T, et al. Probiotics for preventing ventilator-associated pneumonia. Cochrane Database Syst Rev 2014; 10: CD009066.

14 van Ruissen MCE, Bos LD, Dickson RP, et al. Manipulation of the microbiome in critical illness-probiotics as a preventive measure against ventilator-associated pneumonia. Intensive Care Med Exp 2019; 7: 37.

15 Su M, Jia Y, Li Y, et al. Probiotics for the prevention of ventilator-associated pneumonia: a meta-analysis of randomized controlled trials. Respir Care 2020; 65: 673-685.

$16 \mathrm{Gu}$ WJ, Wei CY, Yin RX. Lack of efficacy of probiotics in preventing ventilator-associated pneumonia probiotics for ventilator-associated pneumonia: a systematic review and meta-analysis of randomized controlled trials. Chest 2012; 142: 859-868.

17 Wang J, Liu KX, Ariani F, et al. Probiotics for preventing ventilator-associated pneumonia: a systematic review and meta-analysis of high-quality randomized controlled trials. PLoS ONE 2013; 8: e83934.

18 Kotzampassi K, Giamarellos-Bourboulis EJ, Voudouris A, et al. Benefits of a synbiotic formula (Synbiotic 2000Forte) in critically Ill trauma patients: early results of a randomized controlled trial. World J Surg 2006; 30: $1848-1855$.

19 Higgins JPT, Chandler J, Cumpston M, et al. eds. Cochrane Handbook for Systematic Reviews of Interventions version 6.0 (updated July 2019). https://training.cochrane.org/handbook/archive/v6

20 Liberati A, Moher D, Tetzlaff J, et al. Preferred reporting items for systematic reviews and meta-analyses: the PRISMA statement. PLoS Med 2009; 6: e1000097.

21 Rongrungruang Y. Randomized controlled study of probiotics containing Lactobacillus casei (Shirota strain) for prevention of ventilator-associated pneumonia. J Med Assoc Thai 2015; 98: 253-259.

22 Tan M, Zhu JC, Du J, et al. Effects of probiotics on serum levels of Th1/Th2 cytokine and clinical outcomes in severe traumatic brain-injured patients: a prospective randomized pilot study. Crit Care 2011; 15: R290.

23 Morrow LE, Kollef MH, Casale TB. Probiotic prophylaxis of ventilator-associated pneumonia: a blinded, randomized, controlled trial. Am J Respir Crit Care Med 2010; 182: 1058-1064.

24 Shimizu K, Yamada $\mathrm{T}$, Ogura $\mathrm{H}$, et al. Synbiotics modulate gut microbiota and reduce enteritis and ventilator-associated pneumonia in patients with sepsis: a randomized controlled trial. Crit Care 2018; 22: 239.

25 Zeng J, Wang CT, Zhang FS, et al. Effect of probiotics on the incidence of ventilator-associated pneumonia in critically ill patients: a randomized controlled multicenter trial. Intensive Care Med 2016; 42: 1018-1028.

26 Giamarellos-Bourboulis EJ, Bengmark S, Kanellakopoulou K, et al. Pro- and synbiotics to control inflammation and infection in patients with multiple injuries. J Trauma 2009; 67: 815-821.

27 Knight DJ, Gardiner D, Banks A, et al. Effect of synbiotic therapy on the incidence of ventilator associated pneumonia in critically ill patients: a randomised, double-blind, placebo-controlled trial. Intensive Care Med 2009; 35: 854-861.

28 Barraud D, Blard C, Hein F, et al. Probiotics in the critically ill patient: a double blind, randomized, placebo-controlled trial. Intensive Care Med 2010; 36: 1540-1547.

29 Forestier C, Guelon D, Cluytens V, et al. Oral probiotic and prevention of Pseudomonas aeruginosa infections: a randomized, double-blind, placebo-controlled pilot study in intensive care unit patients. Crit Care 2008; 12: R69.

30 Klarin B, Adolfsson A, Torstensson A, et al. Can probiotics be an alternative to chlorhexidine for oral care in the mechanically ventilated patient? A multicentre, prospective, randomised controlled open trial. Crit Care 2018; 22: 272.

31 Spindler-Vesel A, Bengmark S, Vovk I, et al. Synbiotics, prebiotics, glutamine, or peptide in early enteral nutrition: a randomized study in trauma patients. JPEN J Parenter Enteral Nutr 2007; 31: 119-126.

32 Mahmoodpoor A, Hamishehkar H, Asghari R, et al. Effect of a probiotic preparation on ventilator-associated pneumonia in critically ill patients admitted to the intensive care unit: a prospective double-blind randomized controlled trial. Nutr Clin Pract 2019; 34: 156-162.

33 Li XC, Wang JZ, Liu YH. Effect of probiotics on respiratory tract pathogen colonization in neonates undergoing mechanical ventilation. Chin J Contemp Pediatr 2012; 14: 406-408

34 Banupriya B, Biswal N, Srinivasaraghavan $\mathrm{R}$, et al. Probiotic prophylaxis to prevent ventilator associated pneumonia (VAP) in children on mechanical ventilation: an open-label randomized controlled trial. Intensive Care Med 2015; 41: 677-685.

35 Oudhuis GJ, Bergmans DC, Dormans T, et al. Probiotics versus antibiotic decontamination of the digestive tract: infection and mortality. Intensive Care Med 2011; 37: 110-117.

36 de Jonge E, Schultz MJ, Spanjaard L, et al. Effects of selective decontamination of digestive tract on mortality and acquisition of resistant bacteria in intensive care: a randomised controlled trial. Lancet 2003; 362: 1011-1016.

37 Koeman M, van der Ven AJ, Hak E, et al. Oral decontamination with chlorhexidine reduces the incidence of ventilator-associated pneumonia. Am J Respir Crit Care Med 2006; 173: 1348-1355.

38 Wittekamp BHJ, Plantinga NL, Leleu K, et al. Oral mucosal adverse events with chlorhexidine $2 \%$ mouthwash in ICU. Intensive Care Med 2016; 42: 620-621.

39 Hua F, Xie H, Worthington HV, et al. Oral hygiene care for critically ill patients to prevent ventilator-associated pneumonia. Cochrane Database Syst Rev 2016; 10: CD008367.

40 Giunta V, Ferrer M, Esperatti M, et al. ICU-acquired pneumonia with or without etiologic diagnosis: a comparison of outcomes. Crit Care Med 2013; 41: 2133-2143.

41 Besselink MG, van Santvoort HC, Renooij W, et al. Intestinal barrier dysfunction in a randomized trial of a specific probiotic composition in acute pancreatitis. Ann Surg 2009; 250: 712-719.

42 Fan QL, Yu XM, Liu QX, et al. Synbiotics for prevention of ventilator-associated pneumonia: a probiotics strain-specific network meta-analysis. J Int Med Res 2019; 47: 5349-5374.

43 Cook DJ, Johnstone J, Marshall JC, et al. Probiotics: prevention of severe pneumonia and endotrachea colonization trial-PROSPECT: a pilot trial. Trials 2016; 17: 377.

44 Johnstone J, Heels-Ansdell D, Thabane L, et al. Evaluating probiotics for the prevention of ventilator-associated pneumonia: a randomised placebo-controlled multicentre trial protocol and statistical analysis plan for PROSPECT. BMJ Open 2019; 9: e025228. 
Schuijt TJ, Lankelma JM, Scicluna BP, et al. The gut microbiota plays a protective role in the host defence against pneumococcal pneumonia. Gut 2016; 65: 575-583.

46 Gauguet S, D’Ortona S, Ahnger-Pier K, et al. Intestinal microbiota of mice influences resistance to Staphylococcus aureus pneumonia. Infect Immun 2015; 83: 4003-4014

47 Vieira AT, Rocha VM, Tavares L, et al. Control of Klebsiella pneumoniae pulmonary infection and immunomodulation by oral treatment with the commensal probiotic Bifidobacterium longum 5(1A). Microbes Infect 2016; 18: 180-189.

48 Khailova L, Baird CH, Rush AA, et al. Lactobacillus rhamnosus GG improves outcome in experimental Pseudomonas aeruginosa pneumonia: potential role of regulatory T cells. Shock 2013; 40: 496-503.

49 Tok D, Ilkgul O, Bengmark S, et al. Pretreatment with pro- and synbiotics reduces peritonitis-induced acute lung injury in rats. J Trauma 2007; 62: 880-885.

50 Asahara T, Takahashi A, Yuki N, et al. Protective effect of a synbiotic against multidrug-resistant Acinetobacter baumannii in a murine infection model. Antimicrob Agents Chemother 2016; 60: 3041-3050.

51 Lee YN, Youn HN, Kwon JH, et al. Sublingual administration of Lactobacillus rhamnosus affects respiratory immune responses and facilitates protection against influenza virus infection in mice. Antiviral Res 2013; 98: 284-290.

52 Song JA, Kim HJ, Hong SK, et al. Oral intake of Lactobacillus rhamnosus M21 enhances the survival rate of mice lethally infected with influenza virus. J Microbiol Immunol Infect 2016; 49: 16-23.

53 Percopo CM, Rice TA, Brenner TA, et al. Immunobiotic Lactobacillus administered post-exposure averts the lethal sequelae of respiratory virus infection. Antiviral Res 2015; 121: 109-119.

54 McAleer JP, Kolls JK. Contributions of the intestinal microbiome in lung immunity. Eur J Immunol 2018; 48 39-49.

55 McAleer JP, Nguyen NL, Chen K, et al. Pulmonary Th17 antifungal immunity is regulated by the gut microbiome. I Immunol 2016; 197: 97-107.

56 Besselink MG, van Santvoort HC, Buskens E, et al. Probiotic prophylaxis in predicted severe acute pancreatitis: a randomised, double-blind, placebo-controlled trial. Lancet 2008; 371: 651-659. 